\title{
Effectiveness of the clinical decision support tool ESR eGUIDE for teaching medical students the appropriate selection of imaging tests: randomized cross-over evaluation
}

\author{
Torsten Diekhoff $^{1} \cdot$ Franz Kainberger $^{1} \cdot$ Laura Oleaga $^{1} \cdot$ Marc Dewey $^{1}$ (D) Elke Zimmermann ${ }^{1}$
}

Received: 17 December 2019 / Revised: 17 March 2020 / Accepted: 7 May 2020 / Published online: 20 May 2020

(C) The Author(s) 2020

\begin{abstract}
Objectives To evaluate ESR eGUIDE - the European Society of Radiology (ESR) e-Learning tool for appropriate use of diagnostic imaging modalities - for learning purposes in different clinical scenarios.

Methods This anonymized evaluation was performed after approval of ESR Education on Demand leadership. Forty clinical scenarios were developed in which at least one imaging modality was clinically most appropriate, and the scenarios were divided into sets 1 and 2. These sets were provided to medical students randomly assigned to group A or B to select the most appropriate imaging test for each scenario. Statistical comparisons were made within and across groups.

Results Overall, 40 medical students participated, and 31 medical students (78\%) answered both sets. The number of correctly chosen imaging methods per set in these 31 paired samples was significantly higher when answered with versus without use of ESR eGUIDE $(13.7 \pm 2.6$ questions vs. $12.1 \pm 3.2, p=0.012)$. Among the students in group A, who first answered set 1 without ESR eGUIDE $(11.1 \pm 3.2)$, there was significant improvement when set 2 was answered with ESR eGUIDE $(14.3 \pm 2.5, p=$ 0.013). The number of correct answers in group B did not drop when set 2 was answered without ESR eGUIDE (12.4 \pm 2.6$)$ after having answered set 1 first with ESR eGUIDE $(13.0 \pm 2.7, p=0.66)$.

Conclusion The clinical decision support tool ESR eGUIDE is suitable for training medical students in choosing the best radiological imaging modality in typical scenarios, and its use in teaching radiology can thus be recommended.

Key Points

- ESR eGUIDE improved the number of appropriately selected imaging modalities among medical students.

- This improvement was also seen in the group of students which first selected imaging tests without ESR eGUIDE.

- In the student group which used ESR eGUIDE first, appropriate selection remained stable even without the teaching tool.
\end{abstract}

Keywords Random allocation · Clinical decision support systems $\cdot$ Diagnostic imaging $\cdot$ Learning $\cdot$ Medical students

\section{Abbreviations}

ACR American College of Radiology

ESR European Society of Radiology

Marc Dewey and Elke Zimmermann are equally contributing last authors.

Electronic supplementary material The online version of this article (https://doi.org/10.1007/s00330-020-06942-2) contains supplementary material, which is available to authorized users.

Marc Dewey

marc.dewey@ charite.de

1 Department of Radiology, Charité - Universitätsmedizin Berlin, Humboldt-Universität zu Berlin, Freie Universitat Berlin, Charitéplatz 1, 10117 Berlin, Germany

\section{Introduction}

The Choosing Wisely initiative has identified certain procedures that are not recommended due to low evidence or because they can even be harmful $[1,2]$. However, the initiative's lists were also influenced by political and economic aspects and may thus have limited uptake and influence [3]. Therefore, appropriate-use criteria, ideally developed using evidence-based [4] and Delphi processes [5], hold the greatest potential to address geographical inconsistencies in clinical imaging indications and to reduce the inappropriate conduct of imaging tests while ensuring that necessary imaging tests are done for the right patient at the right point in time [6].

Teaching medical students about the appropriate use of imaging tests could become a key factor in avoiding low- 
value health care and overutilization of diagnostic imaging [7-9]. Especially e-Learning might be suited for interactively teaching medical students the true sense of clinical decision support systems [10] including the Bayesian perspective to diagnostic test selection [11, 12]. The European Society of Radiology (ESR) has developed ESR iGUIDE as a clinical decision support system for appropriate imaging test selection in a variety of clinical scenarios [13]. Recently, this initiative, which became available in 2018, was extended to the teaching of medical students and continuous medical education, and, under the name ESR eGUIDE, was made available as an online electronic learning (e-Learning) source. However, its capability to guide the decision for the appropriate imaging test in a given clinical scenario and to educate medical students have not yet been analyzed.

Therefore, the aim of our study was to test the effectiveness of this e-Learning tool as a clinical decision support in teaching medical students. We hypothesized that ESR eGUIDE might improve the appropriateness of imaging test selection by medical students and thus set up a random cross-over design for evaluation of medical students to test this hypothesis in a variety of clinical scenarios.

\section{Materials and methods}

\section{Study design}

This anonymized study was performed at a single university hospital in Europe (Charité). The study was approved by ESR leadership of the Education on Demand e-Learning group. The study took place as a random cross-over evaluation of medical students in 2017 and 2018 and utilized the first version of the ESR eGUIDE platform (Fig. 1), which transitioned into a new platform at the turn of the year 2018. The ESR eGUIDE allows to review the evidence-based appropriateness of different modalities, and their radiation exposure and costs. The appropriateness is indicated by a score on a 1 to 9 scale (1-3: usually not appropriate, 4-6: may be appropriate, 7-9: usually appropriate) together with an indicator of radiation exposure (0 to 5, 0 : no radiation, $5: 10-30 \mathrm{mSv}$ radiation exposure) and costs of the examination.

\section{Clinical scenarios and test sets}

Forty clinical scenarios were developed by a core team at Charité. In each scenario, at least one imaging modality was clinically most appropriate: angiography, computed tomography, magnetic resonance imaging, x-ray, or sonography. These 40 scenarios were divided into set 1 and set 2 , each including 20 clinical scenarios, and are provided in the Electronic Supplementary Material.

\section{Study conduct}

The two sets of 20 clinical scenarios each were provided to medical students randomly assigned to group A or B to select the most appropriate imaging test for each scenario. Medical student group A first answered set 1 of 20 clinical scenarios without ESR eGUIDE, followed by set 2 with ESR eGUIDE. Medical student group B first answered set 1 of 20 clinical scenarios with ESR eGUIDE, followed by set 2 without ESR eGUIDE.

\section{Study administration}

The purpose of the study was introduced to medical students participating in a voluntary radiology lecture on playful learning imaging test selection ("when and which imaging test to select") and which also explained general principles of the five diagnostic imaging tests. Moreover, the Bayes principle including pre-test probability estimation for test selection was discussed with a handful of clinical examples which were different from those included in the two sets of 20 clinical scenarios. Following this lecture, medical students could enroll in an e-Learning activity which formed this random crossover evaluation study. Enrolled students were randomly assigned to medical student group A or B and received access codes for ESR eGUIDE. Following this registration, students could go through the 40 clinical scenarios included in the two test sets. Students did not know before study conduct to which of the two groups they were assigned and could withdraw from participation at any time. At the end of having answered all scenarios, participants received an analysis of their performance in appropriate diagnostic imaging test selection.

\section{Statistical analysis}

For the prevalence of correct responses, descriptive statistics were used and comparisons were performed using a two-tailed Wilcoxon matched-pairs signed rank. Statistical comparisons were made within and across groups considering a $p$ value below 0.05 as statistically significant. We performed all analysis with the statistical software Prism (Version 8.1; GraphPad).

\section{Results}

\section{Participation}

The number of students invited and those receiving login data is shown in Fig. 2. Overall, 40 medical students participated in this random evaluation of the ESR eGUIDE teaching tool for appropriate medical imaging test selection. Thirty-one of the 
40 medical students (78\%) answered both sets of clinical scenarios (Fig. 2).

\section{Appropriate selection of imaging tests with and without ESR eGUIDE}

The number of appropriately chosen imaging methods per set (mean \pm SD) in the 31 paired samples of the medical student answering both sets of clinical scenarios was significantly higher when answered with $(13.7 \pm 2.6)$ as compared to without $(12.1 \pm 3.2)$ ESR eGUIDE ( $p=0.012$, Fig. 3$)$. Among the students in group A, who answered set 1 without ESR eGUIDE first $(11.1 \pm 3.2)$, there was a significant improvement when set 2 was answered with ESR eGUIDE (14.3 \pm $2.5, p=0.013$, Fig. $4 \mathrm{a}$ ). For both tests, the elimination of one outlier did not change the results significantly ( $p=0.022$ and $p=0.002$, respectively). The number of correct answers in group B did not decline when set 2 was answered without ESR eGUIDE $(12.4 \pm 2.6)$ after having answered set 1 first with ESR eGUIDE $(13.0 \pm 2.7, p=0.66$, Fig. $4 \mathrm{~b})$.

\section{Discussion}

This random cross-over evaluation was conducted to test the effectiveness of ESR eGUIDE to improve the appropriate selection of diagnostic imaging tests by medical students in different clinical scenarios. We found that ESR eGUIDE significantly improved the proportion of appropriately selected imaging tests. This was most likely a result of the better guidance provided by listings of appropriate, indeterminate, and inappropriate imaging tests for certain scenarios. These results are of relevance as they may inform medical training curricula, such as the ESR Curriculum for Undergraduate Radiological Education (https://www.myesr.org/media/229), by putting

\section{Group A and Group B 40 participating students; 31 paired samples}

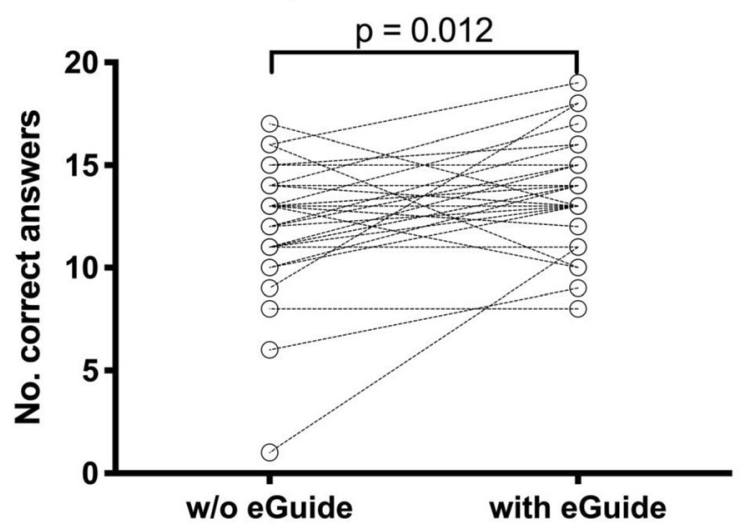

Fig. 3 Significant improvement in questionnaire value $(\min 0, \max 20)$ in the 31 paired samples through the use of ESR eGUIDE $(p=0.012)$ greater weight on e-Learning and radiology in undergraduate teaching [14-16].

We think that this survey has two implications. Firstly, clinical decision support tools are suitable to improve students' knowledge in selecting appropriate diagnostic imaging tests. This may help in disseminating ESR eGUIDE to other institutions using the evidence generated in this study. Secondly, in the student group which used ESR eGUIDE first, similarly high appropriate test ordering was found when this group analyzed the second set of clinical scenarios without ESR eGUIDE. This suggests that sustained skills might develop with the use of ESR eGUIDE and persist even in future settings when clinical decision support systems $[17,18]$ are not at hand anymore.

Digital transformation will also fundamentally change eLearning to reach new levels using image interaction possibilities, which were not included in the present study, yet may further increase radiology knowledge and skills of medical students [19]. Interestingly, e-Learning can also be used to increase skills in optimizing acquisition and reducing artifacts in magnetic resonance imaging [20]. For a long time, eLearning has already been used to assist medical students in understanding anatomy [21]. Including adaptive tutorials in teaching diagnostic test selection principles might further improve the understanding of imaging test properties by medical students. This was not yet included in the present analysis due to the complexity of setting up personalized learning experiences [22]. Whether or not additional teaching of students by radiology residents using web-based educational material may improve clinical decision-making skills is also not clear yet.

In a survey, medical students participating in a radiology clerkship stated that they would be interested in using the appropriateness criteria of the American College of Radiology (ACR) for effective utilization of imaging tests and evidence-based imaging [23]. Another study among third-year medical students, however, showed that use of the ACR appropriate-use criteria was actually low and did not increase after interventions improving familiarity with such criteria [24]. The most important reason for this failure was the lack of a quick, easy-to-use online mobile applicationbased interface [24]. We tried to tackle this barrier by evaluating ESR eGUIDE as an online interactive teaching interface for the appropriate utilization of diagnostic imaging tests.

There is an increasing clinical demand for better training of students in selecting the most appropriate imaging test for the right patients at the right point in time, e.g., using ESR eGUIDE. e-Learning approaches to teaching might be best suited for individually adjusting the learning experience. As a result, students might be put in a position to provide greater value to patient care through more consistent and evidencebased selection of imaging tests that are best suited to individual clinical scenarios $[25,26]$. 
Fig. 4 a, b Change in questionnaire values in group $\mathrm{A}$ (w/o ESR eGUIDE first) and group B (with eGIUDE first) a 27 participating students; 21 paired samples

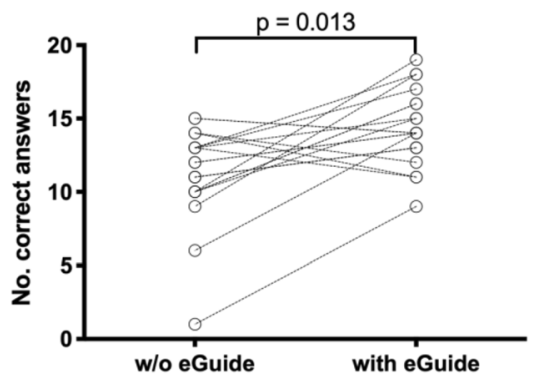

b 13 participating students; 10 paired samples

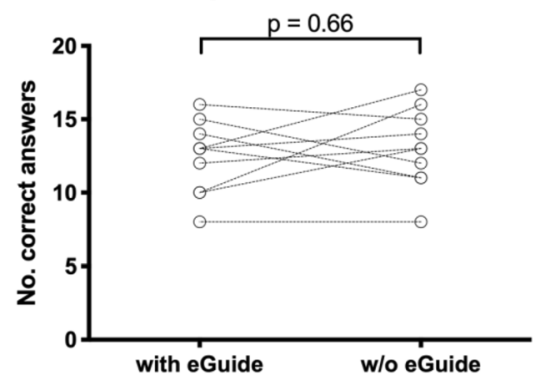

This study is limited by its single-center design and the small number of participating medical students. Especially for the comparisons within group B, the statistics might be underpowered - it might be that no significant differences were found due to the small sample size of 13 participants. Only a fraction of the more than 1000 invited students applied for log-in data (195) and only a fraction of those completed one (40) or both (31) questionnaires. However, we have no information about those who participated nor why the others have decided to not respond to the invitation or to not complete the clinical scenarios. Moreover, it is likely that the participants are more committed than students not participating, which might have biased our results. The random evaluation and cross-over design are advantages that allow balanced comparisons of the effectiveness of ESR eGUIDE. Larger studies in more than one medical university are recommended and appear worthwhile based on the initial favorable results obtained here.

In summary, this random evaluation shows that guiding medical students towards appropriate imaging test selection results in significantly more correctly indicated tests being selected for the individual case scenarios.

Funding information Open Access funding provided by Projekt DEAL. This study has received funding by: Prof. Dewey has received grant support from the Heisenberg Program of the DFG (DE 1361/14-1).

\section{Compliance with ethical standards}

Guarantor The scientific guarantor of this publication is Prof. Dr. Marc Dewey.

Conflict of interest The authors of this manuscript declare relationships with the following companies: Prof. Dewey has received grant support from the FP7 Program of the European Commission for the randomized multicenter DISCHARGE trial (603266-2, HEALTH-2012.2.4.-2). He also received grant support from German Research Foundation (DFG) in the Heisenberg Program (DE 1361/14-1), graduate program on quantitative biomedical imaging (BIOQIC, GRK 2260/1), for fractal analysis of myocardial perfusion (DE 1361/18-1), and the Priority Programme Radiomics (DE 1361/19-1 [428222922] and 20-1 [428223139] in SPP 2177/1). He also received funding from the Berlin University Alliance (GC_SC_PC 27) and from the Digital Health Accelerator of the Berlin Institute of Health. Prof. Dewey has received lecture fees from Canon,
Guerbet. Prof. Dewey is European Society of Radiology (ESR) Research Chair (2019-2022) and the opinions expressed in this article are the author's own and do not represent the view of ESR. Prof. Dewey is also the editor of Cardiac CT, published by Springer Nature, and offers handson courses on CT imaging (www.ct-kurs.de). Institutional master research agreements exist with Siemens, General Electric, Philips, and Canon. The terms of these arrangements are managed by the legal department of Charité-Universitätsmedizin Berlin. Professor Dewey holds a joint patent with Florian Michallek on dynamic perfusion analysis using fractal analysis (PCT/EP2016/071551). Other authors declared no conflicts of interest.

Statistics and biometry One of the authors has significant statistical expertise.

Informed consent Written informed consent was not required for this study because no patients were investigated.

Ethical approval Institutional Review Board approval was not required because no patients were investigated.

\section{Methodology}

- Experimental

- Performed at one institution

Open Access This article is licensed under a Creative Commons Attribution 4.0 International License, which permits use, sharing, adaptation, distribution and reproduction in any medium or format, as long as you give appropriate credit to the original author(s) and the source, provide a link to the Creative Commons licence, and indicate if changes were made. The images or other third party material in this article are included in the article's Creative Commons licence, unless indicated otherwise in a credit line to the material. If material is not included in the article's Creative Commons licence and your intended use is not permitted by statutory regulation or exceeds the permitted use, you will need to obtain permission directly from the copyright holder. To view a copy of this licence, visit http://creativecommons.org/licenses/by/4.0/.

\section{References}

1. Cassel CK, Guest JA (2012) Choosing wisely: helping physicians and patients make smart decisions about their care. JAMA 307 : $1801-1802$

2. Volpp KG, Loewenstein G, Asch DA (2012) Choosing wisely: low-value services, utilization, and patient cost sharing. JAMA $308: 1635-1636$ 
3. Morden NE, Colla CH, Sequist TD, Rosenthal MB (2014) Choosing wisely-the politics and economics of labeling lowvalue services. N Engl J Med 370:589-592

4. Lacson R, Raja AS, Osterbur D et al (2016) Assessing strength of evidence of appropriate use criteria for diagnostic imaging examinations. J Am Med Inform Assoc 23:649-653

5. Taylor AJ, Cerqueira M, Hodgson JM et al (2010) ACCF/SCCT/ ACR/AHA/ASE/ASNC/NASCI/SCAI/SCMR 2010 Appropriate use criteria for cardiac computed tomography. A Report of the American College of Cardiology Foundation Appropriate Use Criteria Task Force, the Society of Cardiovascular Computed Tomography, the American College of Radiology, the American Heart Association, the American Society of Echocardiography, the American Society of Nuclear Cardiology, the North American Society for Cardiovascular Imaging, the Society for Cardiovascular Angiography and Interventions, and the Society for Cardiovascular Magnetic Resonance. J Cardiovasc Comput Tomogr 4:407 e401-407 e433

6. Clauser P, Mann R, Athanasiou A et al (2018) A survey by the European Society of Breast Imaging on the utilisation of breast MRI in clinical practice. Eur Radiol 28:1909-1918

7. Malhotra A, Maughan D, Ansell J et al (2015) Choosing Wisely in the UK: the Academy of Medical Royal Colleges' initiative to reduce the harms of too much medicine. BMJ 350:h2308

8. Glasziou P, Moynihan R, Richards T, Godlee F (2013) Too much medicine; too little care. BMJ 347:f4247

9. Brodersen J, Kramer BS, Macdonald H, Schwartz LM, Woloshin S (2018) Focusing on overdiagnosis as a driver of too much medicine. BMJ 362:k3494

10. Goldzweig CL, Orshansky G, Paige NM et al (2015) Electronic health record-based interventions for improving appropriate diagnostic imaging: a systematic review and meta-analysis. Ann Intern Med 162:557-565

11. Nease RF Jr, Owens DK, Sox HC Jr (1989) Threshold analysis using diagnostic tests with multiple results. Med Decis Making 9: 91-103

12. Sox HC Jr (1986) Probability theory in the use of diagnostic tests. An introduction to critical study of the literature. Ann Intern Med 104:60-66

13. European Society of Radiology (ESR) (2017) Summary of the proceedings of the international forum 2016: "imaging referral guidelines and clinical decision support - how can radiologists implement imaging referral guidelines in clinical routine?'. Insights Imaging 8: $1-9$

14. Nyhsen CM, Steinberg LJ, O'Connell JE (2013) Undergraduate radiology teaching from the student's perspective. Insights Imaging 4:103-109
15. Oris E, Verstraete K, Valcke M, ESR Working Group on Undergraduate Education (2012) Results of a survey by the European Society of Radiology (ESR): undergraduate radiology education in Europe-influences of a modern teaching approach. Insights Imaging 3:121-130

16. European Society of Radiology (ESR) (2011) Undergraduate education in radiology. A white paper by the European Society of Radiology. Insights Imaging 2:363-374

17. Benndorf M, Kotter E, Langer M, Herda C, Wu Y, Burnside ES (2015) Development of an online, publicly accessible naive Bayesian decision support tool for mammographic mass lesions based on the American College of Radiology (ACR) BI-RADS lexicon. Eur Radiol 25:1768-1775

18. Foldyna B, Szilveszter B, Scholtz JE, Banerji D, Maurovich-Horvat P, Hoffmann U (2018) CAD-RADS - a new clinical decision support tool for coronary computed tomography angiography. Eur Radiol 28:1365-1372

19. den Harder AM, Frijlingh M, Ravesloot CJ, Oosterbaan AE, van der Gijp A (2016) The importance of human-computer interaction in radiology E-learning. J Digit Imaging 29:195-205

20. Alsharif W, Davis M, Rainford L, Cradock A, McGee A (2018) Validation of the educational effectiveness of a mobile learning app to improve knowledge about MR image quality optimisation and artefact reduction. Insights Imaging 9:721-730

21. Webb AL, Choi S (2014) Interactive radiological anatomy eLearning solution for first year medical students: development, integration, and impact on learning. Anat Sci Educ 7:350-360

22. Wong V, Smith AJ, Hawkins NJ et al (2015) Adaptive tutorials versus web-based resources in radiology: a mixed methods comparison of efficacy and student engagement. Acad Radiol 22:12991307

23. Dillon JE, Slanetz PJ (2010) Teaching evidence-based imaging in the radiology clerkship using the ACR appropriateness criteria. Acad Radiol 17:912-916

24. Kozak B, Webb EM, Khan BK, Orozco NM, Straus CM, Naeger DM (2015) Medical student usage of the American College of Radiology Appropriateness Criteria. Acad Radiol 22:1606-1611

25. Dewey M (2018) The future of radiology: adding value to clinical care. Lancet 392:472-473

26. European Society of Radiology (ESR) (2017) ESR concept paper on value-based radiology. Insights Imaging 8:447-454

Publisher's note Springer Nature remains neutral with regard to jurisdictional claims in published maps and institutional affiliations. 